Henri J. Vial
Marie-Laure Ancelin

\title{
Les lipides de l'érythrocyte impaludé. Applications pharmacologiques
}

\begin{abstract}
Le métabolisme lipidique des plasmodies est intense, complexe et d'une importance vitale pour le parasite. Expérimentalement, les composés qui interfèrent avec ce métabolisme sont toxiques pour le parasite. Il se pourrait donc que ce type de produit se révélât d'une grande importance dans la chimiothérapie antipaludique future et constituât de ce fait l'une des réponses à la chimiorésistance croissante du parasite.
\end{abstract}

\section{TIRÉS A PART}

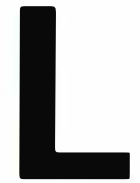

e futur de la chimiothérapie antipaludique est particulièrement alarmant au vu des résistances croisées des parasites à des médicaments qui ne sont par ailleurs pas reliés structuralement. Une large extension de cette polypharmacorésistance est maintenant inévitable. Seules des études détaillées sur la biologie et la biochimie de ce parasite permettront l'élaboration de nouvelles stratégies chimiothérapeutiques. L'accumulation intra-érythrocytaire d'une quantité considérable de phospholipides nécessaire à la biogenèse des membranes de Plasmodium constitue l'une des propriétés les plus intrigantes du parasite. Les recherches dans ce domaine permettent d'entrevoir à terme une approche originale pour une nouvelle chimiothérapie du paludisme.

En effet, la prolifération asexuée intra-érythrocytaire du parasite (phase associée aux symptômes cliniques de la maladie) s'accompagne d'une néosynthèse d'une quantité considérable de membranes. Si les membranes du parasite contiennent peu ou pas de cholestérol, en revanche, l'augmentation en phospholipides de l'érythrocyte, après impaludation, atteint $500 \%$ lorsque le parasite est au stade mature. Ceci opère au sein de l'érythrocyte mature de mammifère, qui ne possède qu'un faible renouvellement de ses písuspholipides et qui en aucun cas n'a la capacité de biosynthèse des phospholipides ou des acides gras. Ce métabolisme phospholipidique est donc effectué par la seule machinerie enzymatique du parasite.

\section{Biosynthèse des phospholipides dans la cellule impaludée}

Les phospholipides du parasite sont synthétisés à partir d'unités de base essentielles puisées dans le plasma, têtes polaires et acides gras, que le parasite est bien incapable de synthétiser. Dès nos premières études [1], nous avons mis en évidence des voies métaboliques auparavant infirmées (phosphatidyléthanolamine-N-méthylase) ou non détectées (phosphatidylsérine-décarboxylase) permettant aujourd'hui de dresser un schéma quasi complet de ce métabolisme au sein de Plasmodium (voir [2] pour revue).

Phosphatidylcholine (PC) et phosphatidyléthanolamine (PE) sont les phospholipides majeurs du parasite, représentant ensemble plus de $85 \%$ des phospholipides totaux. Le parasite possède deux moyens pour se procurer le $\mathrm{PE}$ (biosynthèse de novo et décarboxylation de phosphatidylsérine [PS]), tout comme pour synthétiser la PC (voie de novo et méthylation de $\mathrm{PE}$ ) (figure 1). Un équilibre entre les différentes voies métaboliques aboutissant au même phospholipide est en partie effectif (phéno- 


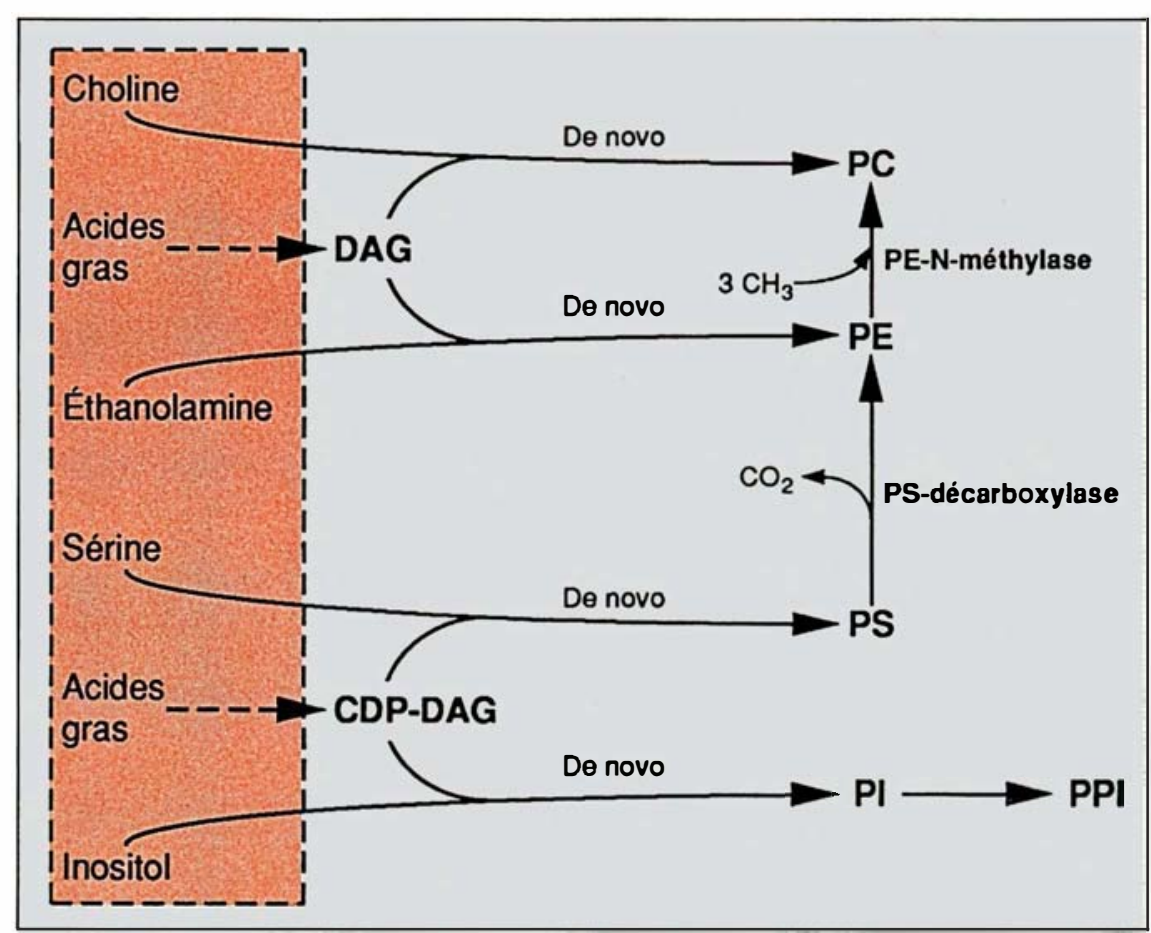

Figure 1. Schéma simplifié du métabolisme phospholipidique dans la cellule impaludée. Les unités de base puisées dans le plasma sont en grisé dans le cadre en pointillé. $D A G=$ diacylglycérols ; $P C, P E, P I, P S=$ phosphatidylcholine, -éthanolamine, -inositol, -sérine; PPI = polyphospho-inositides.

mène de compensation), et l'existence de pools différents pour un même phospholipide à l'intérieur du parasite est tout à fait plausible.

La présence du cycle complet des polyphospho-inositides au sein même de Plasmodium est maintenant assurée. Ignorant l'activateur naturel de ce cycle, nous avons notamment montré une libération d'inositol trisphosphate par un subterfuge utilisant un ionophore du calcium pour activer la phospholipase $\mathrm{C}$ intracellulaire, spécifique $\mathrm{du}$ phosphatidylinositol-4,5-bisphosphate. Une hydrolyse des phosphoinositides semble opérer à l'étape d'exflagellation au cours de la formation de l'œuf [3]. Ces données sont probablement d'un grand intérêt pour la physiologie de ce parasite.

\section{Approches pharmacologiques}

Ce travail, essentiellement fonda$\mathrm{m} / \mathrm{s} n^{\circ} 10$ vol. 5 , décembre 89 un effet cholinergique. Plus de 70 de ces composés ont été testés et l'effet antipaludéen in vitro est parfaitement corrélé à la capacité d'inhiber sélectivement la synthèse des phospholipides. Les tests d'activité sur des isolats chloroquine sensibles ou polypharmacorésistants de $P$. falciparum n'ont pas présenté de différences. Enfin, des acides gras modifiés stoppent la croissance in vitro de Plasmodium, le pouvoir inhibiteur étant corrélé à l'inhibition de l'acylCoA synthétase, première enzyme prenant en charge les acides gras pour leur incorporation dans les phospholipides [6].

Le métabolisme phospholipidique se prête donc bien à des interférences pharmacologiques. C'est un modèle pharmacologique nouveau que nous développons dans le cadre d'une sélection de molécules ayant un mode d'action original, espérant ainsi retarder l'apparition de résistances. La concrétisation à terme par un médicament est cependant retardée par une pauvreté remarquable dans la pharmacologie des phospholipides, y compris pour les lipides pharmacologiquement actifs.

\section{Structure fine de la membrane plasmique de l'érythrocyte impaludé}

mental au départ, s'est vite révélé porteur d'une application possible pour une nouvelle chimiothérapie du paludisme : le métabolisme phospholipidique est nécessaire à la maturation intracellulaire du parasite et toute altération de ce métabolisme conduit irrémédiablement à la mort du parasite. Ainsi, des aminoalcools proches de l'éthanolamine stoppent la croissance de $P$. falciparum in vitro. Ces composés conduisent à la formation de phospholipides nouveaux (faux métabolites), ce qui altère en retour la biosynthèse des phospholipides naturels [4]. Des molécules plus volumineuses, analogues potentiels de la choline, bloquent le transporteur de choline de l'érythrocyte infecté, ce qui interdit toute synthèse de PC et provoque la mort du parasite. Les concentrations inhibitrices $50 \%$ sur la croissance du parasite atteignent $5.10^{-7}[5]$. Ces molécules n'induisent pas de faux métabolites mais certaines possèdent
$\mathrm{Au}$ cours de son développement intra-érythrocytaire, le parasite établit des structures de liaison avec l'érythrocyte hôte, produisant des changements dans la membrane érythrocytaire afin d'assurer sa nutrition et sa survie [2, 7].

Avec un laboratoire hollandais de l'université d'Utrecht, nous avons montré que la distribution asymétrique des phospholipides de la membrane érythrocytaire n'était pas modifiée, infirmant un rôle direct de PS dans la physiopathologie du paludisme [8] (l'apparition du phospholipide anionique, PS, dans le feuillet externe des membranes plasmiques est connue pour entraîner une activation de la voie alterne du complément et provoquer une interaction de la cellule avec l'endothélium des capillaires et avec les macrophages).

En revanche, les vitesses de mobilité transverse (flip) des phospholipides au sein de cette membrane sont 
RÉFÉRENCES

l. Vial H, Thuet M, Broussal J, Philippot J. Phospholipid biosynthesis by Plasmodium knowlesi-infected erythrocytes: the incorporation of phospholipid precursors and the identification of previously undetected metabolic pathways. J Parasitol 1982; 68 : 379-91.

2. VialHJ, Ancelin ML, Philippot JR, Thuet MJ. Biosynthesis and dynamics of lipids in Plasmodium-infected mature mam. malian erythrocytes. Blood Cells 1990 (sous presse).

3. Pett M, Martin S, Schneider I. Phosphoinositide hydrolysis in $P$. falciparum malaria. FASEB J 1989 ; 3 : 17371

4. Vial HJ, Thuet MJ, Ancelin ML, Philippot JR, Chavis C. Phospholipid metabolism as a new target for malaria chemotherapy. Mechanism of action of D-2-amino-1-butanol. Biochem Pharmacol 1984; 33 : 2761-70.

5. Ancelin ML, Vial HJ. Quaternary ammonium compounds efficiently inhibit Plasmodium falciparum growth in vitro by impairment of choline transport. Antimicrob Agents Chemother 1986 ; 29 : 814-20.

6. Beaumelle BD, Vial $\mathrm{HJ}$. Correlation of the efficiency of fatty acid derivatives in suppressing Plasmodium falciparum growth in culture with their inhibitory effect on acyl-CoA synthetase activity. Mol Biochem Parasitol $1988 ; 28$ : 39-42.

7. Howard R J. Malarial proteins at the mem brane of Plasmodium falciparum-infected erythrocytes and their involvement in cytoadherence to endothelial cells. Prog Allergy $1988 ; 41$ : 98-147

8. Moll GN, Vial HJ, Bevers EM, et al: Phospholipid asymmetry in the plasma membrane of malaria infected erythrocytes. Biochem Cell Biol 1990 (sous presse)

9. Beaumelle $\mathrm{BD}, \mathrm{Vial} \mathrm{HJ}$, Bienvenüe $\mathrm{A}$. Enhanced transbilayer mobility of phospholipids in malaria-infected monkey erythrocytes. A spin label study. J Cell Physiol 1988 $135: 94-100$

10. Haldar K, De Amorim AF, Cross GAM Transport of fluorescent phospholipid analogues from the erythrocyte membrane to the parasite in Plasmodium falciparum infected erythrocytes. J Cell Biol 1989; 108 : 2183-92.

11. Ginsburg H, Stein D. New permeability pathways induced by the malarial parasite in the membrane of its host erythrocyte : potential routes for targeting of drugs into infected cells. Biosc Rep 1987; 7 : 455-63.

12. Schlesinger PH, Krogstad DJ, Herwaldt BL. Antimalarial agents : mechanisms of action. Antimicrob Agents Chemother considérablement augmentées [9, 10]. Cela pourrait faire partie de modifications plus larges conduisant à un passage des phospholipides du plasma vers le parasite. Le développement du parasite paraît également associé à des changements dans les espèces moléculaires de $\mathrm{PC}$ et $\mathrm{PE}$ au sein de la membrane érythrocytaire, sans cependant observer de modification dans la répartition des classes de phospholipides [2]. Cela témoigne d'une dynamique intense des phospholipides dans la cellule impaludée et apparaît comme la première preuve expérimentale de la capacité du parasite à manipuler la composition lipidique de son hôte. Ces modifications peuvent expliquer les changements de fluidité ou de pression de surface observés dans l'érythrocyte après infection et semblent d'ailleurs la cause de la destruction sélective des érythrocytes infectés (qui présentent un degré de compression des phospholipides différent de celui des érythrocytes sains) par une phospholipase $\mathrm{A}_{2}$ modifiée par acylation [2]. Ainsi, les modifications de propriétés de la membrane érythrocytaire après infection peuvent être à la base d'une reconnaissance sélective des érythrocytes infectés, par des enzymes, ou à terme par des drogues [11].

De tels résultats contribuent à préciser la physiologie de ce parasite intracellulaire et la physiopathologie du paludisme. Ce programme s'inscrit dans le cadre plus large de recherches approfondies et récentes sur la biochimie de Plasmodium afin de découvrir de nouvelles pistes aboutissant à une pharmacologie dirigée [12], condition nécessaire au contrôle de la transmission du paludisme et de ses complications potentiellement létales, et à l'évaluation de nouveaux traitements contre $P$. falciparum résistant...

\section{H.-J. Vial chargé de recherche à l'Inserm. M.-L. Ancelin chargée de recherche à l'Inserm. Cnrs URA 530, USTL, CP 107, 34095 Montpellier Cedex 2, France.}

\section{Summary}

Lipids and the malarial parasite

The dramatic resurgence of malaria calls for original therapeutic approaches. One consists of interferring with parasite-specific metabolisms. This single-cell eukaryote is bewilderingly versatile in the synthesis of phospholipids that accumulate in its membranes, and seems to be as intricate as many multicellular organisms. This metabolism has been identified as a potential target because of its intensity, specificity and vital importance. Compounds that interfere with the supply of plasmatic precursors (polar heads or fatty acids) or with their metabolism are lethal to the parasite. Malaria infection is also associated with modifications in the lipid complement of the host erythrocyte membrane, which could explain changes in fluidity and membrane properties. Basically, knowledge concerning metabolisms specific to the parasite can provide a wide range of targets for research in rational drug design programmes.
Remerciements

Nos recherches sont soutenues par l'OMS (UNDP/World Bank/WHO-TDR programme), l'INSERM, et la Commission des Communautés européennes. 
ERRATUM. Nous republions dans ce numéro les figures 1 et 3 de l'article de Claudine Junien et Isabelle Henry : «Bras court du chromosome 11 : empreinte parentale, tumorigenèse et pertes d'allèles » (m/s $\mathrm{n}^{\circ} 7$, vol. 5, p. 480, septembre 1989).

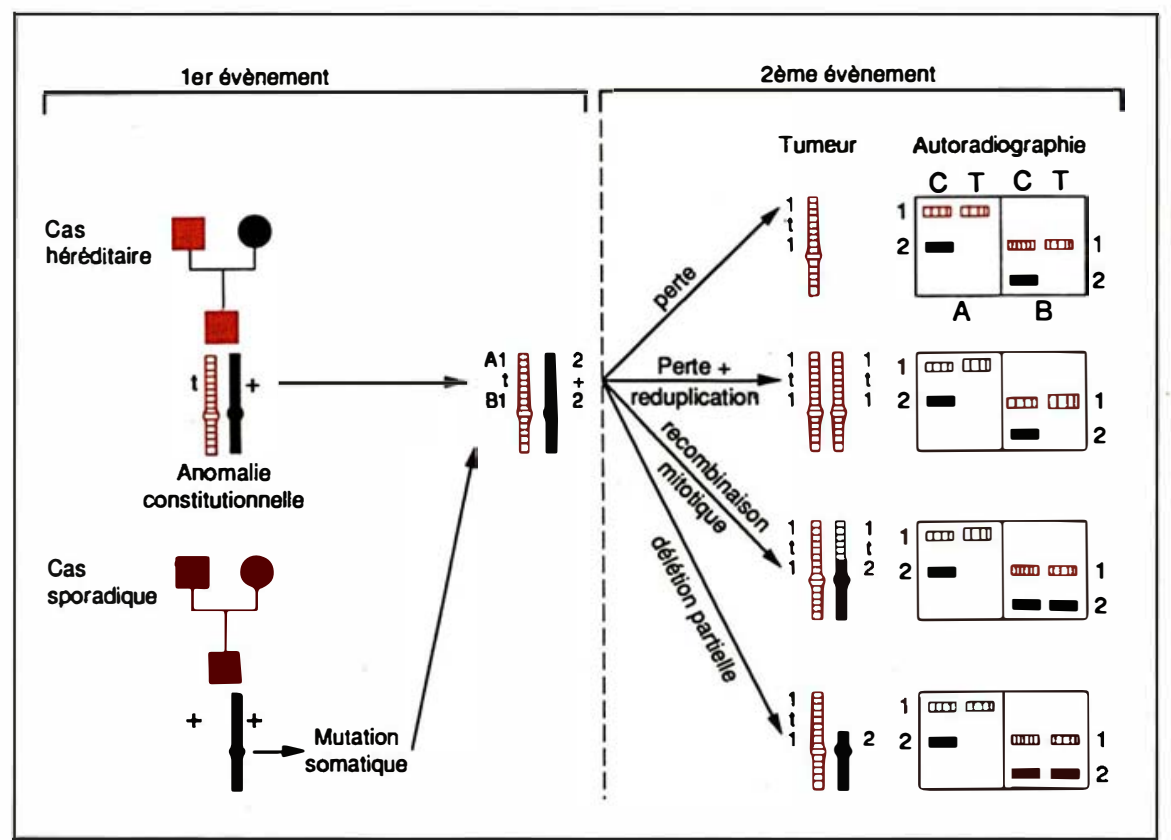

Figure 1. "Pertes d'allèles" ou "perte de l'hétérozygotie" dues à des remaniements chromosomiques somatiques. La comparaison des âges d'apparition de tumeurs de la rétine à caractère héréditaire et de tumeurs sporadiques a permis à Knudson de proposer la « théorie des deux événements ». Dans les tumeurs héréditaires, une première mutation d'origine germinale est présente dans toutes les cellules de l'individu. II suffit qu'une deuxième mutation, somatique cette fois, se produise pour que la tumeur apparaisse. Dans les tumeurs sporadiques, il faudra que la deuxième mutation somatique se produise dans une cellule dans laquelle une première mutation se sera déjà produite. Représentation schématique des différents mécanismes impliqués dans la perte de l'hétérozygotie, montrant la comparaison des génotypes constitutionnels et tumoraux par la technique de Southern, pour les polymorphismes étudiés. $t=$ allèle altéré $;+=$ allèle sauvage; $A$ et $B=$ séquences $A D N$ détectant des polymorphismes (RFLP), choisies de telle sorte que l'individi soit hétérozygote. 1 et 2 $=$ allèles des différents marqueurs. $C=A D N$ constitutionnel provenant des lymphocytes du sujet. $T=A D N$ tumoral. En noir : sont représentés les chromosomes portant un allèle normal. En rouge : sont représentés les chromosomes portant un allèle muté. Dans l'exemple choisi, l'allèle muté est d'origine paternelle, dans les formes sporadiques et familiales. En fait, dans les formes familiales, il pourrait aussi bien être d'origine maternelle.

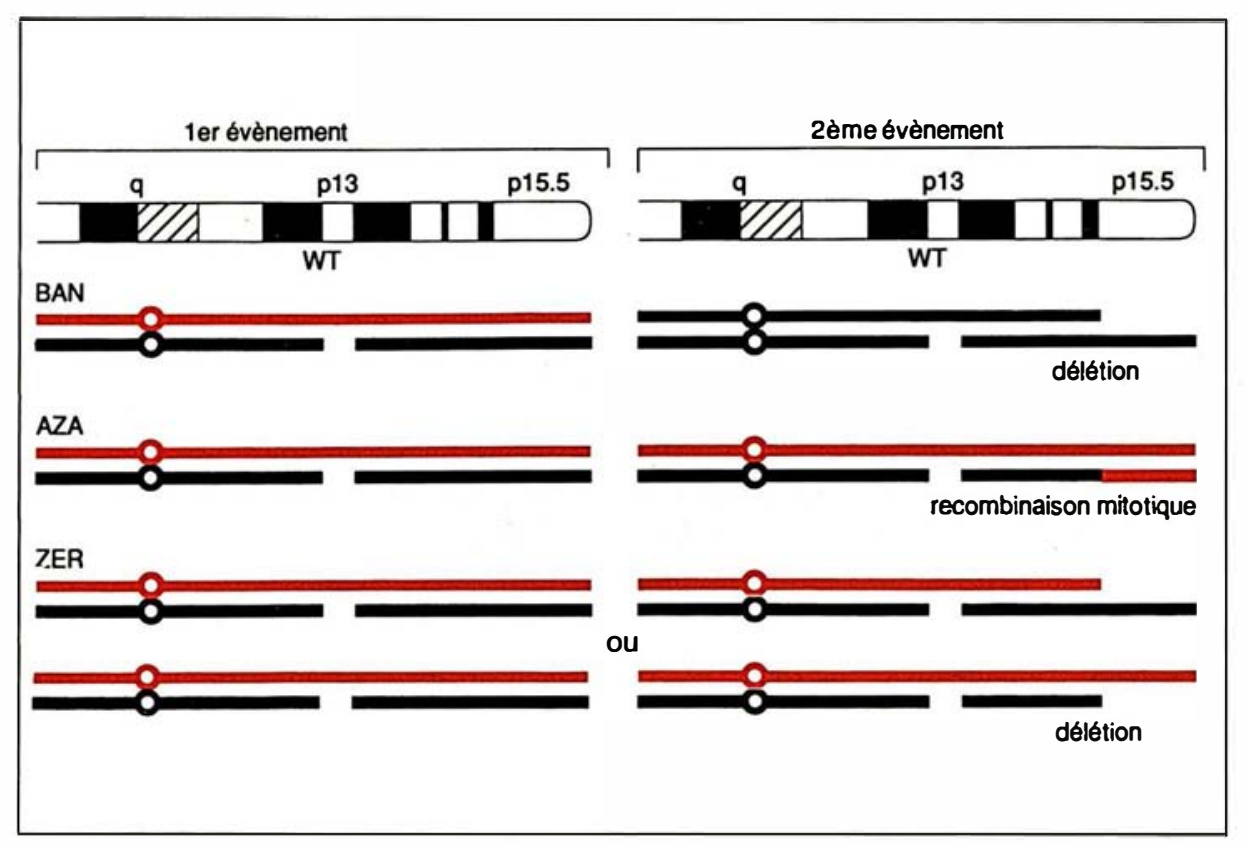

$m / s n^{o} 10$ vol. 5 , décembre 89
Figure 3. Identification et caractérisation des remaniements constitutionnels (1 or événement) et acquis $\left(2^{\circ}\right.$ événement) dans trois cas de syndrome WAGR ltumeur de Wilms, aniridie, anomalies génito-urinaires, arriération mentalel avec délétion constitutionnelle en $11 \mathrm{p} 13 \mathrm{et}$ perte d'allèles en 11 p 15.5 dans la tumeur. Dans le premier cas (BAN), la réalisation d'hybrides somatiques a permis de montrer que la perte d'allèles, due à une délétion en 11p15.5, touchait le chromosome normal non porteur de la délétion constitutionnelle en 11p13 [21]. Dans le deuxième cas (AZA), l'étude familiale a montré que la délétion constitutionnelle en 11p13 ainsi que la perte d'allèles en 11p15.5, due ici à une recombinaison mitotique, touchait le même chromosome, le chromosome d'origine maternelle. Dans le troisième cas (ZER), en l'absence d'hybrides somatiques et d'étude familiale, il n'a pas été possible d'identifier l'origine du chromosome ayant subi les remaniements. 